\title{
PulseRider Treated Aneurysm with Significant Artifact on Postoperative Magnetic Resonance Angiography: A Case Report and Literature Review
}

\author{
Anthony V. Nguyen, $\mathrm{MD}^{1,2}$, Laura K. Reed, $\mathrm{MD}^{1,2}$, Walter S. Lesley, MD, MBA ${ }^{1,2}$ \\ ${ }^{1}$ Department of Neurosurgery, Baylor Scott \& White Medical Center, Temple, TX, USA \\ ${ }^{2}$ Department of Surgery, Texas A\&M University College of Medicine, Temple, TX, USA
}

The PulseRider is a neuroendovascular adjunct for wide-necked intracranial aneurysms. The decreased metal burden of the PulseRider theoretically reduces artifact on radiologic imaging. However, we report here on a case of a patient who underwent PulseRider-assisted stent-coiling of a basilar tip aneurysm. He returned 19 months later for intermittent diplopia and darkening of vision but was neurologically intact on exam. Both contrast-enhanced and time-offlight magnetic resonance angiography (MRA) demonstrated absence of signal in the basilar artery in the proximal anchors of the PulseRider. Given his lack of reproducible symptoms and high functional status, it is presumed that the imaging reflected artifact and not thrombosis/ stenosis. Although the PulseRider is a useful treatment option for wide-necked intracranial aneurysms, the clinician should be aware that even contrast-enhanced MRA can produce artifact that resembles thrombosis/stenosis. Non-angiogram radiologic imaging modalities may be appropriate for evaluation for residual aneurysm but not patency of the parent artery.

Key Words: PulseRider; Intracranial aneurysm; Stent-assisted coiling; Neuroendovascular surgery; Neurosurgery

\section{INTRODUCTION}

Wide-necked intracranial aneurysms are traditionally treated by open microsurgical clipping, primary or stent-assisted coiling, or balloon remodeling. A number of different treatments have been developed to accommodate variations in anatomy. A risk of traditional coiling of wide-necked aneurysms is migration of the coils back into the parent artery, and traditional stent-assisted coiling of basilar tip aneurysms is not optimal due to the relationship of the aneurysm to the posterior cerebral arteries. Stent-assisted coiling of basilar tip aneurysms has been described with placing two stents in a Y-shaped configuration or by placing a horizontally oriented stent, but the technical difficulty of these approaches has incentivized the design of adjunct devices such as the PulseRider.

The PulseRider (Cerenovus, Miami, FL, USA) is a neuroendovascular adjunct that allows for a variation on stent-assisted coiling. It is mostly made of nitinol with platinum/iridium alloy radiopaque markers and also contains stainless steel at the proximal detachment points. The PulseRider can treat bifurcation aneu-

\section{Correspondence to:} Anthony V. Nguyen, MD Department of Neurosurgery, Baylor Scott \& White Medical Center, 2401 S 31st Street MS-01-610A, Temple, TX USA

Tel: +1-713-478-0518

Fax: +1-254-724-5779

E-mail:avn1401@bswhealth.org

Received: May 17, 2021

Revised: July 25, 2021

Accepted: July 26, 2021
Copyright $\odot 2021$ Korean Society of Interventional Neuroradiology This is an Open Access article distributed under the terms of the Creative Commons Attribution Non-Commercial License (http://creativecommons.org/licenses/by-nc/4.0) which permits unrestricted non-commercial use, distribution, and reproduction in any medium, provided the original work is properly cited. 
rysms that have a branch artery angle of up to 120 degrees relative to the parent artery. Endovascular treatment of widenecked cerebral aneurysms with the PulseRider has a high technical success rate, and there is complete occlusion of approximately two-thirds of aneurysms at one-year after treatment and adequate occlusion of $90 \%$ at one-year. ${ }^{2,3}$ Additionally, the PulseRider is composed of less metal than a traditional stent with larger fenestrations, which may allow for easier coiling.

Follow-up of patients who received the PulseRider can be conducted with conventional cerebral digital subtraction angiography (DSA), magnetic resonance angiography (MRA), or computed tomography angiography (CTA) per the Federal Drug Administration (FDA) instructions for use. We report on the case of a contrast-enhanced MRA (CE-MRA) of a PulseRider-treated patient demonstrating focal absent flow signal at the proximal end of the PulseRider due to device artifact.
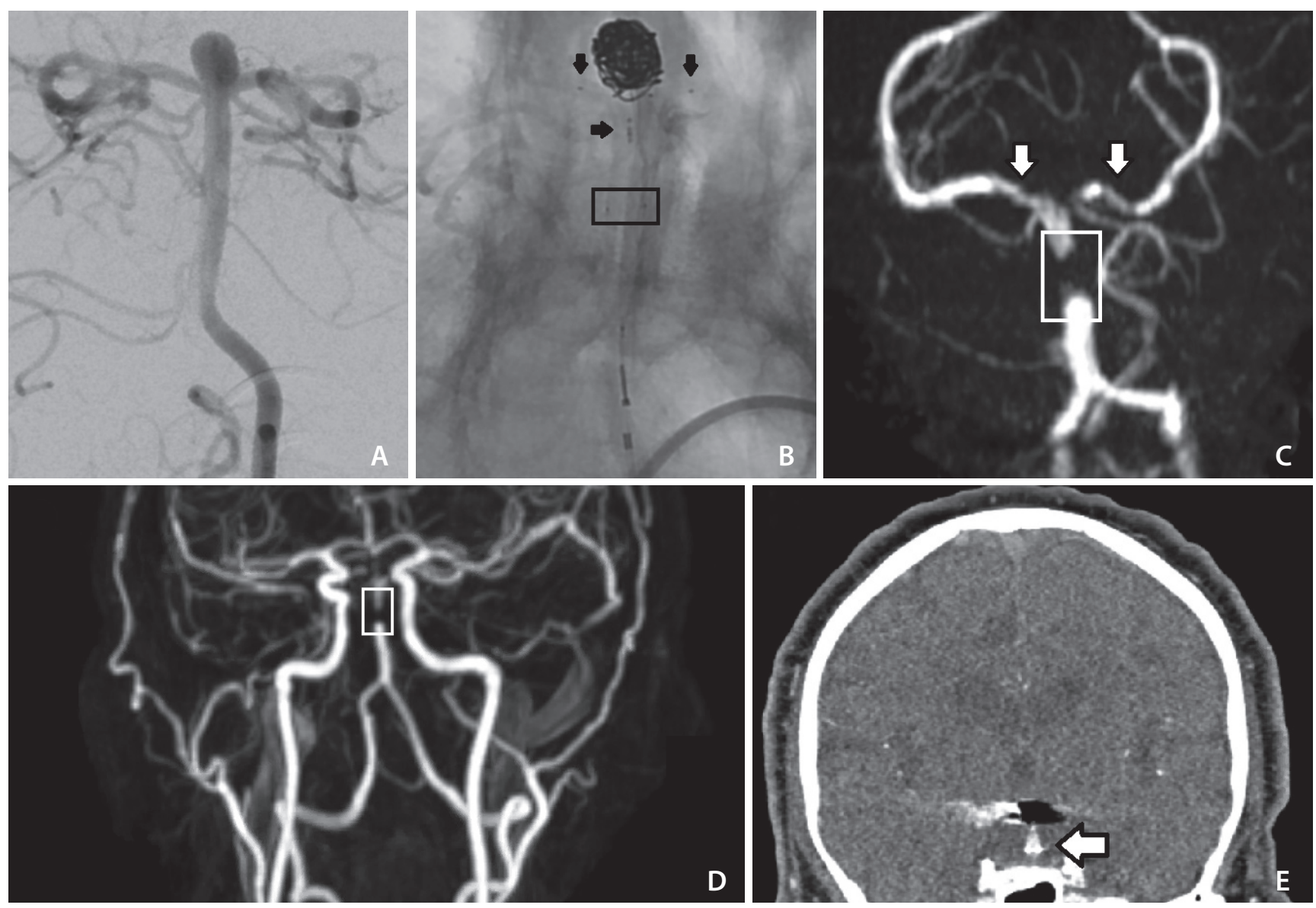

Fig. 1. Diagnostic cerebral angiogram demonstrating wide-necked basilar tip aneurysm (A). Diagnostic cerebral angiogram demonstrating patent basilar artery terminating in the bilateral posterior cerebral arteries status-post PulseRider-assisted coiling (B). Black horizontal arrows denote the medial markers of the PulseRider residing in the basilar artery, and black vertical arrows denote the markers of the PulseRider residing in the posterior cerebral arteries. A black box denotes the proximal radio-opaque markers of the PulseRider where the stainless-steel proximal anchors reside. Magnetic resonance angiography (MRA) of the head utilizing time-of-flight (TOF) techniques without contrast (C) and of the neck with contrast-enhanced techniques (D). In TOF-MRA, there is complete loss of signal at the proximal portion of the PulseRider (white box) and decreased but not absent signal within the proximal P1 segments of the bilateral posterior cerebral arteries (white arrows outlined in black). In CE-MRA, the artifact is again demonstrated in the proximal portion of the PulseRider (white box). There is signal distally without filling of the aneurysm. These results indicate that flow of the basilar artery to the posterior cerebral artery is not compromised and that there is no residual or recurrent aneurysm. A coronal reconstruction of computed tomographic angiography (CTA) demonstrates significant coil artifact and also two foci of PulseRider metal artifact resembling (white arrow outlined in black) in the proximal anchoring portion of the PulseRider (E). The stainless-steel retained in the proximal anchors are likely the culprit of the MRA and CTA artifact. 


\section{CASE REPORT}

An elderly patient presented to clinic for neurosurgical evaluation of an expanding basilar tip aneurysm. The aneurysm measured $7 \mathrm{~mm}$ in height with a neck of $4 \mathrm{~mm}$ in width (Fig. 1A). Given the location and wide-necked configuration of the aneurysm, endovascular treatment was favored, and traditional coiling would not be sufficient. After discussion of risks and benefits, the patient elected to proceed with PulseRider-assisted coiling. A 211-D (parent vessel diameter 2.7-3.5 mm) T-shaped PulseRider was successfully deployed with the T-struts well-aligned to the P1 segments of the posterior cerebral arteries (Fig. 1B). The patient was discharged within 24 hours with instructions to take aspirin $81 \mathrm{mg}$ and clopidogrel $75 \mathrm{mg}$ daily. The patient did not return for scheduled postoperative clinic; however, at 19-months following surgery he returned to his primary physician for episodic diplopia (including monocular vision diplopia) and intermittent darkening of vision that occurred approximately twice per month.

CTA head obtained at that time was unremarkable, although the study was degraded by metallic streak artifact from the PulseRider and coils. He was referred back to our clinic for evaluation. At 20-months after surgery, time-offlight MRA (TOF-MRA) of the head and CE-MRA of the neck (both 3 Tesla, echo time 22, echo train length 27, repetition time 700 , flip angle 120) were performed. Both the modalities demonstrated lack of signal within the junction of the middle and distal thirds of the basilar artery trunk, in the vicinity of the detachment zone of the PulseRider device (Fig. 1C, D). However, both imaging modalities demonstrated flow within the distal basilar artery at the bifurcation to the posterior cerebral arteries. Thus, the artifact was limited to the anchors. Of note, there was no evidence of residual or recurrent aneurysm. Comparison against the patient's prior CTA demonstrated that this segment of the basilar artery produced significant metal artifact (Fig. 1E). The patient's symptoms were not reproducible, and he was neurologically intact. Given the patient's reassuring neurologic exam and the imaging findings, it was determined that the aneurysm was successfully treated and that the loss-of-signal was artifact. The vision complaints prompting this re-evaluation at 20-months were not due the treated aneurysm or the surgery. DSA was offered to the patient to rule out absence of in-stent stenosis or evaluate for other possible cerebrovascular causes of the patient's symptoms, but the patient declined the procedure.

\section{DISCUSSION}

In this case report, we describe and demonstrate CE-MRA artifact due to the PulseRider device. The patient was followed up with a CE-MRA that demonstrated a focal loss of both TOF and contrast-enhanced flow signal along a portion of the proximal margin of the PulseRider, raising concern for device-related vessel stenosis or thrombus formation. The PulseRider FDA instructions for use state that DSA, MRA, or CTA are appropriate modalities for follow-up. Large multicenter studies have allowed for the treating surgeon to follow with their imaging modality of choice. ${ }^{2,3}$ This case report implies that MRA is appropriate for follow-up aneurysms but not necessarily the parent vessel.

A literature review of the PubMed Central database was performed to review cases of PulseRider-treated follow-up imaging. This was performed by utilizing the search terms ("PulseRider" OR "Pulse Rider"). The literature review returned 44 works. Excluding conference poster and oral presentations, 37 articles were evaluated. All articles were then reviewed for demonstration or mention of artifact on follow-up MRA after PulseRider treatment of intracranial aneurysms. One study reported artifact and non-visualization of the parent artery with TOF-MRA. ${ }^{4}$ Another study demonstrated similar artifact on MRA but does not specify the exact MRA modality. ${ }^{5}$ However, the authors of this study do cite another study that investigates optimization of TOF-MRA when mentioning this artifact. While Mukherjee et al. ${ }^{6}$ mention that follow-up magnetic resonance imaging demonstrates stent artifact, they do not specify the exact modality or demonstrate the artifact, and their statement implies that magnetic resonance modalities cannot be utilized to evaluate the aneurysm itself. They do not mention evaluation of the parent vessel. No study demonstrated loss of signal with CE-MRA.

CE-MRA has been compared to TOF-MRA, with DSA as the gold standard comparison, for evaluation of aneurysms following treatment with Pipeline Embolization Device, coils, and stent-assisted coils. ${ }^{7-9}$ CE-MRA has been demonstrated to better visualize stented arteries, reduce coil artifact, and more accurately measure luminal diameter. CE-MRA correlates better with DSA than TOF-MRA. ${ }^{10}$ Outside of the literature review, non-visualization of a parent artery in a PulseRider-treated patient on TOF-MRA was corrected with CE-MRA."

This is the first description in conjunction with radiograph- 
ic depiction of a focal artifact in the proximal portion of the PulseRider device observed on CE-MRA. This is likely due to the stainless steel that is retained at the detachment points as the "marker band effect" previously described in the literature for nitinol Neuroform (Stryker, Fremont, CA, USA) stents occurred at vessels less than $2 \mathrm{~mm}$ thick, and the Neuroform markers lie in a plane perpendicular to the flow of blood whereas the PulseRider markers are parallel. ${ }^{12}$ Stainless steel, on the other hand, has been demonstrated to cause complete loss of signal even in a stent that is $8 \mathrm{~mm}$ thick. $^{13}$ The artifact demonstrated here does not hinder evaluation of residual or recurrent aneurysm as there is distal reconstitution of signal after a short segment of artifact. The PulseRider remains a useful adjunct, but clinicians should be aware of this possibility on postoperative imaging.

Although this work is limited in that it is a single case report, we believe it is important for neuroendovascular surgeons to be aware that focal loss of signal within the parent artery can radiographically appear as an in-stent stenosis or thrombosis and this artifact can persist even with CE-MRA in patients with the PulseRider. Thus, while MRA may be a useful imaging modality for assessment of residual aneurysm, it may be limited in assessment of the patency of the parent artery. While there is the remote possibility this patient did indeed have an in-stent stenosis, this is considered unlikely given that his symptoms had a functional overlay component, and he had no persistent neurologic deficits on exam. However, this is a possible limitation of this case report. The patient followed up with physicians outside of our system, so to our knowledge there is no established diagnosis for the patient's symptoms. However, given that he was not referred back to us, there was presumably not an identified cerebrovascular cause for the patient's symptoms. Further studies will be necessary to determine how frequently this artifact occurs, especially considering the overall lower metal burden of the PulseRider.

The PulseRider is a newer FDA-approved adjunctive device that can be used for the treatment of wide-necked intracranial aneurysms. Follow-up imaging of patients who undergo endovascular treatment with the PulseRider can be conducted by DSA, MRA, or CTA. Although CE-MRA has been demonstrated to be better than TOF-MRA for follow-up of aneurysms treated with devices with metal artifact, CE-MRA may still fail to resolve artifact produced in the parent artery of a wide-necked aneurysm treated by PulseRider-assisted coiling and thus may be most appropriate for evaluation for residual aneurysm but not patency of the parent artery.

\section{Fund}

None.

\section{Ethics Statement}

This study was reviewed by our Institutional Review Board and conducted in concordance with IRB policies. The IRBs of Baylor Scott and White Health and Texas A\&M University have waived the requirement for informed consent. Since the consent for publication was not available for the patients mentioned in the figures, patient's information was anonymized by removing the sex and specific age.

\section{Conflicts of Interest}

The authors have no conflicts to disclose.

\section{Author Contributions}

Concept and design: AVN, LKR, and WSL. Analysis and interpretation: AVN, LKR, and WSL. Data collection: AVN. Writing the article: AVN, LKR, and WSL. Critical revision of the article: AVN, LKR, and WSL. Final approval of the article: AVN, LKR, and WSL. Overall responsibility: AVN and WSL.

\section{ORCID}

Anthony V. Nguyen: https://orcid.org/0000-0002-3566-2659

Laura K. Reed: https://orcid.org/0000-0002-7226-2179

Walter S. Lesley: https://orcid.org/0000-0003-4612-4781

\section{REFERENCES}

1. Hendricks BK, Yoon JS, Yaeger K, Kellner CP, Mocco J, De Leacy RA, et al. Wide-neck aneurysms: systematic review of the neurosurgical literature with a focus on definition and clinical implications. J Neurosurg 2020;133:159-165

2. Spiotta AM, Chaudry MI, Turner RD 4th, Turk AS, Derdeyn CP, Mocco J, et al. An update on the adjunctive neurovascular support of wide-neck aneurysm embolization and reconstruction trial: 1-year safety and angiographic results. AJNR Am J Neuroradiol 2018;39:848-851

3. Srinivasan VM, Srivatsan A, Spiotta AM, Hendricks BK, Ducruet $A F$, Albuquerque $F C$, et al. Early postmarket results with PulseRider for treatment of wide-necked intracranial aneurysms: a multicenter experience. J Neurosurg 2020;133:1756-1765

4. Sakai N, Imamura H, Arimura K, Funatsu T, Beppu M, Suzuki K, et 
al. PulseRider-assisted coil embolization for treatment of intracranial bifurcation aneurysms: a single-center case series with 24-month follow-up. World Neurosurg 2019;128:e461-e467

5. Gory B, Spiotta AM, Mangiafico S, Consoli A, Biondi A, Pomero E, et al. PulseRider stent-assisted coiling of wide-neck bifurcation aneurysms: periprocedural results in an international series. AJNR Am J Neuroradiol 2016;37:130-135

6. Mukherjee S, Chandran A, Gopinathan A, Putharan M, Goddard T, Eldridge PR, et al. PulseRider-assisted treatment of widenecked intracranial bifurcation aneurysms: safety and feasibility study. J Neurosurg 2017;127:61-68

7. Boddu SR, Tong FC, Dehkharghani S, Dion JE, Saindane AM. Contrast-enhanced time-resolved MRA for follow-up of intracranial aneurysms treated with the pipeline embolization device. AJNR Am J Neuroradio/ 2014;35:2112-2118

8. Anzalone N, Scomazzoni F, Cirillo M, Righi C, Simionato F, Cadioli $\mathrm{M}$, et al. Follow-up of coiled cerebral aneurysms at 3T: comparison of 3D time-of-flight MR angiography and contrast-en- hanced MR angiography. AJNR Am J Neuroradiol 2008;29:15301536

9. Choi JW, Roh HG, Moon WJ, Kim NR, Moon SG, Kang CH, et al. Time-resolved 3D contrast-enhanced MRA on 3.0T: a non-invasive follow-up technique after stent-assisted coil embolization of the intracranial aneurysm. Korean J Radio/ 2011;12:662-670

10. Levent A, Yuce I, Eren S, Ozyigit O, Kantarci M. Contrast-enhanced and time-of-flight MR angiographic assessment of endovascular coiled intracranial aneurysms at 1.5 T. Interv Neuroradiol 2014;20:686-692

11. De Leacy R, Yaniv G, Nael K. Cerebral aneurysm follow-up: how standards have changed and why. Endovasc Today 2019;18:80-84

12. Agid R, Schaaf M, Farb R. CE-MRA for follow-up of aneurysms post stent-assisted coiling. Interv Neuroradiol 2012;18:275-283

13. Wang Y, Truong TN, Yen C, Bilecen D, Watts R, Trost DW, et al. Quantitative evaluation of susceptibility and shielding effects of nitinol, platinum, cobalt-alloy, and stainless steel stents. Magn Reson Med 2003;49:972-976 